\title{
'Deprescribing' antipsychotics in schizophrenia: witless and dangerous?
}

\section{Ann Mortimer}

\section{COMMENTARY ON... DEPRESCRIBING ANTIPSYCHOTICS ${ }^{\dagger}$}

\begin{abstract}
SUMMARY
'Deprescribing' here comprises arguments against treating patients who have been psychotic beyond first episode. Nevertheless, four out of five patients relapse, compared with only two of five still taking medication. However, much first-episode psychosis is not schizophrenia, but persistent substance-induced psychotic disorder. Virtually nothing is known about factors which facilitate this in the minority who develop it, nor how to decide if the patient will relapse off medication. Patients who are well, stable and tolerating minimal doses of medication should be the first to suggest that they try to do without it, not us.
\end{abstract}

\section{DECLARATION OF INTEREST}

A.M. has worked with pharmaceutical companies regarding the introduction of atypical antipsychotic treatments for schizophrenia, received research funding and spoken at conferences.

\section{KEYWORDS}

deprescribing; risk; Antipsychotics; Polypharmacy; Psychotic Disorders; Schizophrenia; Biological

Treatments; Community.

'Deprescribing', as originated, applies to patients taking numbers of medications for a variety of disorders. To 'home in' on a single class attaching to one disorder surely amounts to no more than clinical common sense, the obvious good practice of establishing the lowest possible maintenance dose, minimising side-effects while preventing relapse. This is not new.

It is conceded that established guidelines mandate long-term treatment in established schizophrenia, since the vast majority of the evidence from discontinuation, 'drug holiday' and ultra-low-dose studies over many years points in the direction of substantially worse relapse rates compared with maintenance treatment. Surely if antipsychotics cannot be dispensed with in the shorter term, there is little logic for doubting their longer-term effectiveness. One could say the same about insulin in diabetes.

\section{Examining the evidence}

The results of discontinuation (Gupta 2018) are well exemplified by the Wunderink trial in remitted first-episode psychosis, which they cite. Only one in five discontinuers did not relapse, albeit in the long term having a better recovery than those maintained on medication. A simple explanation is that these one in five had less severe psychotic disorder than the other four, so recovered better whether they were treated or not. Relapse in discontinuers was double that in the maintenance group, and functional outcome worse. Furthermore, Chen et al's previous randomised controlled trial (Chen 2010), which Gupta et al rather strangely do not cite, recruited first-episode patients without active symptoms, maintained on antipsychotics for at least a year: expected to have a good outcome, discontinuation was a realistic option for these patients. Patients took $400 \mathrm{mg}$ daily quetiapine or placebo. A year later, four of five $(79 \%)$ of the placebo group had relapsed, a result virtually identical to Wunderink et al's, versus two of five (41\%) on quetiapine. Therefore, any recommendation that a patient with first-episode psychosis, never mind established schizophrenia, should cease antipsychotic medication, especially if this is not their idea, is reminiscent of Lord Farquaad's remark in Shrek (2001): 'Some of you may die, but it's a sacrifice I am willing to make'.

\section{Not all psychosis is schizophrenia}

Diagnostic clarity is, however, crucial to appropriate treatment: not all psychosis turns out to be schizophrenia. Early intervention studies report falling case/non-case ratios among patients with firstepisode psychosis (O`Donoghue 2012). In my own service, approaching half of new patients have persistent substance-induced psychotic disorder (Mortimer 2015). Although patients may later attract a schizophrenia-spectrum diagnosis (NiemiPynttar 2013), there is a flaw in any contention that such patients really had schizophrenia all the
Ann Mortimer is emeritus professor at the University of Hull, UK; consultant psychiatrist at NAViGO; and chief medical officer at TranQuality Solutions. She trained in psychiatry in Yorkshire prior to becoming a senior lecturer at Imperial College London, UK. Professor Mortimer took up the foundation chair in Psychiatry at Hull in 1996.

Correspondence Ann Mortimer, NAViGO House, 3-7 Brighowgate, Grimsby DN32 OOE, UK.

Email: ann.mortimer@nhs.net

Copyright and usage

(C) The Royal College of Psychiatrists 2018

${ }^{\dagger}$ See pp. 295-302, this issue. 
time. Early chronic substance misuse in vulnerable individuals may cause subtle brain damage that is not recoverable, even after several years of antipsychotic treatment. This is not the same thing as having schizophrenia in the first place. Clinically, such patients usually have suffered significant adversity, which perhaps explains why they develop psychosis when, fortunately, the vast majority of young substance misusers survive without it. There is very little, if any, research into these important matters going on at the moment: some such patients remain fully recovered without maintenance treatment, particularly if abstinent, but many do not. There is nothing, currently, to inform deprescribing decisions for these patients.

\section{To whose benefit, whose risk?}

Returning to an analysis of the risks and benefits of deprescribing, it is worth pointing out that not every patient develops problematic long-term side-effects. In any case, patients' lifestyle choices confound these associations. Generic drugs are cheap, but relapse is very expensive: psychosocial interventions are not inexpensive, either.

Gupta and colleagues describe a very cumbersome process of deprescribing, with overinclusive involvement of just about everybody. Fine statements about the dignity of risk and the right to fail are all very well, except that the pieces have to be picked up, by somebody, afterwards. Relapse is unpleasant and costly to all concerned: accrual of residual positive, negative and cognitive symptoms comprises further risk. The human costs of suicide and homicide perpetrated by untreated patients, although mercifully uncommon, are disastrous, leaving aside the public resources implicated in such events.

Gupta and colleagues themselves concede that fewer than four out of ten patients desire an active role in their treatment. Many patients and their families want a recommendation, not to have responsibility for clinical management decisions at least partly foisted upon them. The corollary of this is that if anything goes wrong, it is not the fault of the clinician, because the patient signed up to the decision. This can too easily be seen as an abdication of responsibility, no matter how many mental health professionals involved themselves in deprescribing decisions which did not emanate from the patient's own wishes. Social psychology says that people need assistance if they lack the ability, the inclination or both, to decide for themselves: perhaps this is what the six out of ten patients are saying. The 'good old days' of 'Me doctor, you idiot' and 'When we want your opinion, we'll tell you what it is' (!) should be well and truly over, not replaced with ideologically driven blanket treatment strategies outwith the patient's choosing.

In our current state of knowledge, which is that who will relapse and who will not cannot be predicted, there is a consequential question regarding whether the medication-free status and better functional outcome of one in five patients can be justified by the relapses of the other four. For patients considered well and stable, and tolerating minimal effective medication, the consideration of deprescribing antipsychotic treatment cannot but introduce unnecessary risk.

\section{References}

Chen EHY, Lam MML, Chiu CPY, et al (2010) Maintenance treatment with quetiapine versus discontinuation after one year of treatment in patients with remitted first episode psychosis: randomised controlled trial. BMJ, 341: $c 4024$.

Gupta S, Cahill JD, Miller R (2018) Deprescribing antipsychotics: a guide for clinicians. BJPsych Advances, 24(5): 295-302.

Mortimer A (2015) Early intervention in psychosis: another triumph of hope over experience? Progress in Neurology and Psychiatry, 19(3): 10-4

Niemi-Pynttar JA, Sund R, Putkonen H, et al (2013) Substance-induced psychoses converting into schizophrenia: a register-based study of 18,478 Finnish inpatient cases. Journal of Clinical Psychiatry, 74(1): 94-9.

O'Donoghue B, Lyne J, Renwick L (2012) A descriptive study of 'non cases' and referral-rates to an early intervention for psychosis service. Early Interventions in Psychiatry, 6(3): 276-82.

Shrek (2001) produced by PDI/Dreamworks, USA. Directed by Andrew Adamson and Vicky Jenson. [Film]. 